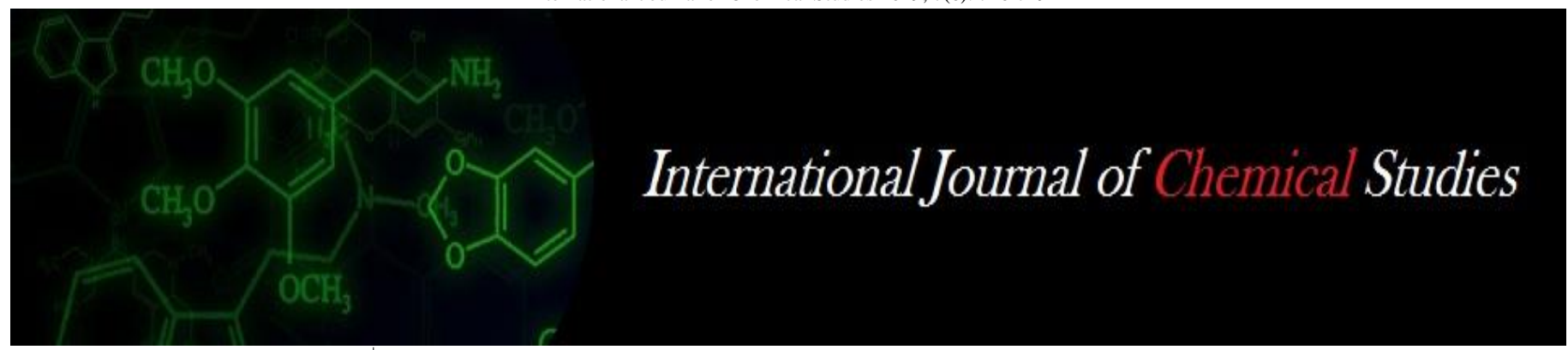

P-ISSN: 2349-8528

E-ISSN: 2321-4902

IJCS 2019; 7(6): 726-729

(C) 2019 IJCS

Received: 07-09-2019

Accepted: 09-10-2019

Yadav Sunil Machindra PG Scholar, Department of Poultry Science Madras Veterinary College Tamil Nadu Veterinary and Animal Sciences University, Chennai, Tamil Nadu, India

\section{Kanagaraju $P$}

Assistant Professor, Department of Poultry Science Madras

Veterinary College, Tamil Nadu Veterinary and Animal Sciences University, Chennai, Tamil

Nadu, India

Srinivasan G

Professor and Head Department of Poultry Science Madras Veterinary College Tamil Nadu Veterinary and Animal Sciences University, Chennai, Tamil

Nadu, India

\section{Rathnapraba $\mathbf{S}$}

Associate Professor, Vaccine Research Centre- Viral Vaccines Tamil Nadu Veterinary and Animal Sciences University Madhavaram Milk Colony, Chennai, Tamil Nadu, India

\section{Survase Swapnil}

PG Scholar, Department of Poultry Science Madras

Veterinary College Tamil Nadu Veterinary and Animal Sciences University, Chennai, Tamil

Nadu, India

\section{Corresponding Author:}

Kanagaraju $P$

Assistant Professor, Department of Poultry Science Madras

Veterinary College, Tamil Nadu Veterinary and Animal Sciences

University, Chennai, Tamil

Nadu, India

\section{Synthesis and characterization of feed grade nano dicalcium phosphate for poultry feeding}

\author{
Yadav Sunil Machindra, Kanagaraju P, Srinivasan G, Rathnapraba S and \\ Survase Swapnil
}

\begin{abstract}
Dicalcium phosphate nanoparticles (nano-DCP) were synthesized by physical method using ball mill. The synthesized DCP nanoparticles were characterized by various techniques viz. Particle size analyser, Zeta potential, Transmission Electron Microscopy (TEM), X-ray diffraction (XRD), Fourier Transform Infrared Spectroscopy (FTIR) and Energy Dispersive Analysis by X-ray (EDAX). The results revealed that synthesized nano-DCP had particle size between $10-20 \mathrm{~nm}$ and zeta potential value of $-25.6 \pm 2.2 \mathrm{mV}$. Crystal size of nano DCP analysed by XRD was $29.31 \mathrm{~nm}$. Calcium and phosphorus content of synthesized nano-DCP were 23.22 and 17.67 per cent. Ball milling, a popular physical method and is easy to operate, economic, time saving and higher yield. Based on its bioavailability, the synthesized feed grade nano DCP can be utilized for in ovo feeding of broiler embryos, livestock and poultry feeding.
\end{abstract}

Keywords: Nano dicalcium phosphate, synthesis, characterization, ball mill, poultry

\section{Introduction}

Calcium and phosphorus are involved in many vital biological activities of the chicken. Requirement of such nutrient is more in case of poultry to perform these activities (Hassan et al., 2016) ${ }^{[1]}$. Use of costly inorganic source of phosphorus in poultry production results in increase of feed cost which in turn cost of production and also leads to excess amount of excretion in the droppings which leads to wastage of nutrients and negative effect on environment.

Nanotechnology, new emerging era of poultry nutrition becoming a popular trend because of its versatile advantages like increased bioavailability, absorption and reduction in excreta and its minimum quantity required in feed compared to conventional sources (Swain et al., 2015) ${ }^{[2]}$. Dicalcium phosphate (DCP) is a dibasic calcium phosphate commonly used as a feed supplement and also as an important source of calcium and phosphorus for livestock and poultry.

Calcium phosphate nanoparticles can be synthesized by various method viz. physical (Ramesh, 2014) ${ }^{[3]}$, wet chemical (Huang et al., 2004) ${ }^{[4]}$, solid state (Ota and Iwashita, 1998) ${ }^{[5]}$, sol-gel (Samy et al., 2015) [6], mechanochemical (Nakamura et al., 2001) [7] and microwave processing (Sarig and Kahana, 2002) ${ }^{[8]}$ methods. Out of these, physical method is more suitable because of its advantages viz., simple to operate, low cost of production and the possibility to scale it to produce large quantities (McCormick,1995) ${ }^{[9]}$.

Hence, an attempt has been made in this study to synthesize the nano dicalcium phosphate particles by using ball milling method to assess its suitability for livestock and poultry feeding.

\section{Materials and Methods}

Feed grade dicalcium phosphate powder (DCP) was purchased from the Centre for Advanced Studies in Poultry Science, College of Veterinary and Animal Sciences, Kerala Veterinary and Animal Sciences University, Mannuthy, Thrissur, India.

\section{Synthesis of nano dicalcium phosphate}

Nano particles of DCP were prepared by physical method using ball mill and it was synthesised by grinding feed grade bone based dicalcium Phosphate in a ball mill (8000D Mixer/Mill - Dual High Energy Ball Mill) at a rotatory speed of 1060 cycles/min using two $12.7 \mathrm{~mm}$ stainless steel ball in each jar of $75 \mathrm{ml}$ capacity for 60 minutes. 
Characterization of nano dicalcium phosphate particles

Synthesized nano dicalcium Phosphate particles were characterized by different techniques for confirmation of its nanostructure and functional properties.

\section{Particle size analyser and Zeta potential}

Average particle size $(\mathrm{nm})$ and Zeta potential $(\mathrm{mV})$ of nanoDCP particles were determined based on the principle of photon correlation spectroscopy using Particle Size Analyser (HORIBA Scientific Nano partica SZ-100). Samples were diluted with MilliQ water and after 2 minutes of sonication, measurements were taken at a scattering angle of $90^{\circ}$ and at a temperature of $25{ }^{\circ} \mathrm{C}$. Samples were taken in disposable plastic cuvette for particle size analysis and electrode cell for zeta potential.

\section{Transmission Electron microscopy (TEM)}

The shape and size of dicalcium phosphate nanoparticles were analysed by using Transmission Electron Microscopy (TEM). Samples were prepared by mixing $1 \%$ phosphotunstic acid with aqueous dispersion of nano particles on a para film. A copper grid was placed over the surface of the liquids. After drying of the copper grid in an incubator and it was examined under a transmission electron microscope and the image for taken to analyse its size and shape.

\section{X-ray diffraction (XRD)}

Structural aspects of prepared nano form of dicalcium phosphate were determined by X-Ray Diffraction technique using Rigaku Mini Flex-II Desktop X-Ray Diffractometer as per the protocol explained by Cengiz et al. (2008) ${ }^{[10]}$. The mean crystal size of particles was calculated from Scherrer's equation (Azaroff, 1968) ${ }^{[11]}$.

\section{Fourier Transform Infrared Spectroscopy (FTIR)}

The surface chemistry of the synthesised nano form of dicalcium phosphate was investigated by Fourier Transform Infra-Red (FTIR) spectroscopy (Agilent Model Cary 630). The FTIR spectra of the samples were obtained in the spectral range of $4000-400 \mathrm{~cm}^{-1}$.

\section{Energy Dispersive Analysis by X-ray (EDAX)}

The percentage of calcium and phosphate present in prepared nano form of dicalcium Phosphate was estimated using Energy Dispersive Analysis by X-ray, using scanning electron microscope (EDAX: SUPRA 55-CARL ZEISS, Germany).

\section{Results and Discussion}

Nano dicalcium phosphate $47.5 \mathrm{~g} / \mathrm{hr}$ was produced from $50 \mathrm{~g}$ of dicalcium phosphate coarse particles using ball mill method. The production efficiency of this method was 95 per cent. The results of the present study was confirmed by the findings of Ramesh, (2014) [3] and Singh and Karmakar, (2011) [12] who reported that optimum quantity of nano particles can be produced by physical method using ball mill.

\section{Particle size analyser and Zeta potential}

The results of particle size analyser and zeta potential revealed that synthesized nanoparticle had average particle size of $62.6 \pm 3.4 \mathrm{~nm}$ (Fig.1) and zeta potential of $-25.6 \pm 2.2$ $\mathrm{mV}$ (Fig.2). This range clearly indicated the uniform distribution of particles in the sample. Ramesh (2014) [3] reported that the nano form of dicalcium phopshtae can be produced by ball milling with an average diameter of 46.60 $\mathrm{nm}$ and confirmed by characterization under particle size analyser. Zeta potential value of nano-DCP $(-25.6 \pm 2.2 \mathrm{mV})$ obtained in this study was in agreement with Nanocomposix, (2012) ${ }^{[13]}$ which explained that zeta potential values greater than $+25 \mathrm{mV}$ or less than $-25 \mathrm{mV}$ typically have high degree of stability.

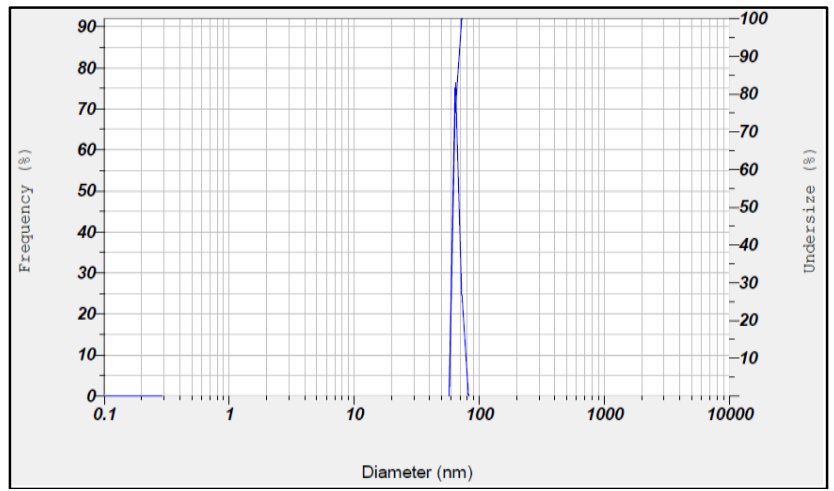

Fig 1: Size distribution pattern of nano-DCP

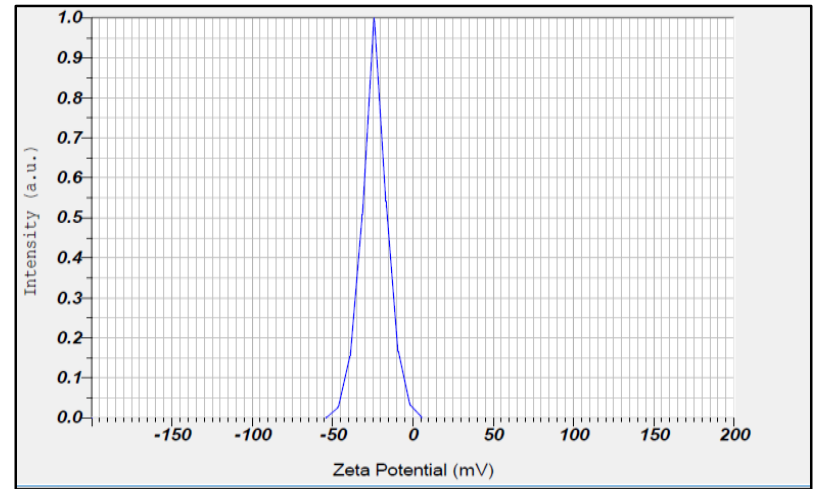

Fig 2: Zeta potential value of nano-DCP

\section{Transmission Electron microscopy (TEM)}

The size of the nano DCP particles analysed through TEM (Fig.3) revealed that nano-DCP powder contain finer particles of 10-20 nm in size and spherical in shape. Similar results were observed by Welzel et al. (2004) [14]; Sokolova et al. (2006) ${ }^{[15]}$, Qing et al. (2012) ${ }^{[16]}$ and Ramesh, (2014) ${ }^{[3]}$ who reported spherical shape nano calcium phosphate particles with particle size between 20-30 nm under TEM. Whereas, Xiaochen et al. (2012) [17] observed rod shaped nano hydroxyapatite by transmission electron microscopy with average particle size of $23 \pm 5 \mathrm{~nm}$.

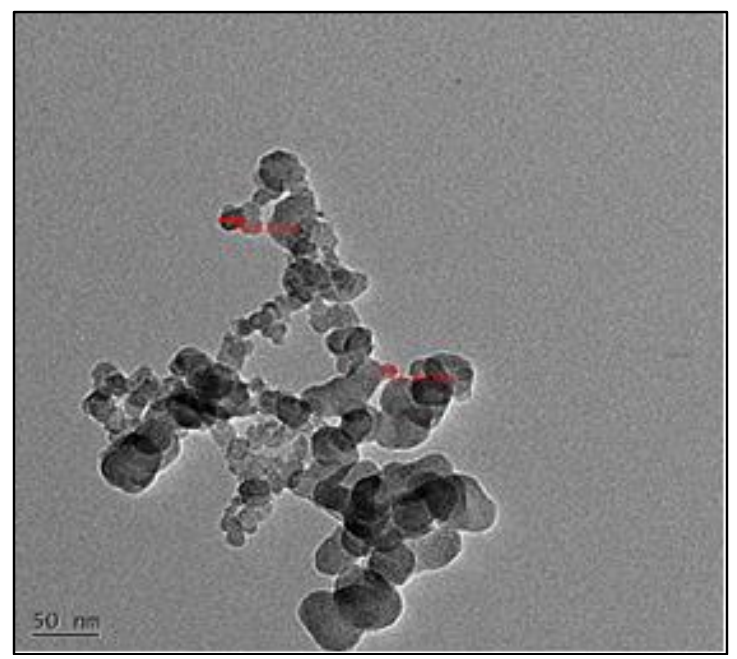

Fig 3: Image of nano DCP under transmission electron microscopy 


\section{X-ray diffraction (XRD)}

The XRD pattern (Fig.4) of nano-DCP particles revealed that the synthesized nano particles are crystalline in nature and matches very well with that of the standard dicalcium phosphate inorganic particles. The data recorded in the $2 \theta$ was analysed using Jade 6.0 software and the average crystallite size obtained was $29.31 \mathrm{~nm}$. Similar findings were reported by Singh and Karmakar (2011) ${ }^{[12]}$ and Cengiz et al. (2008) ${ }^{[10]}$ who observed crystal size of $21 \mathrm{~nm}$ and 15.88 to $16.12 \mathrm{~nm}$ respectively.

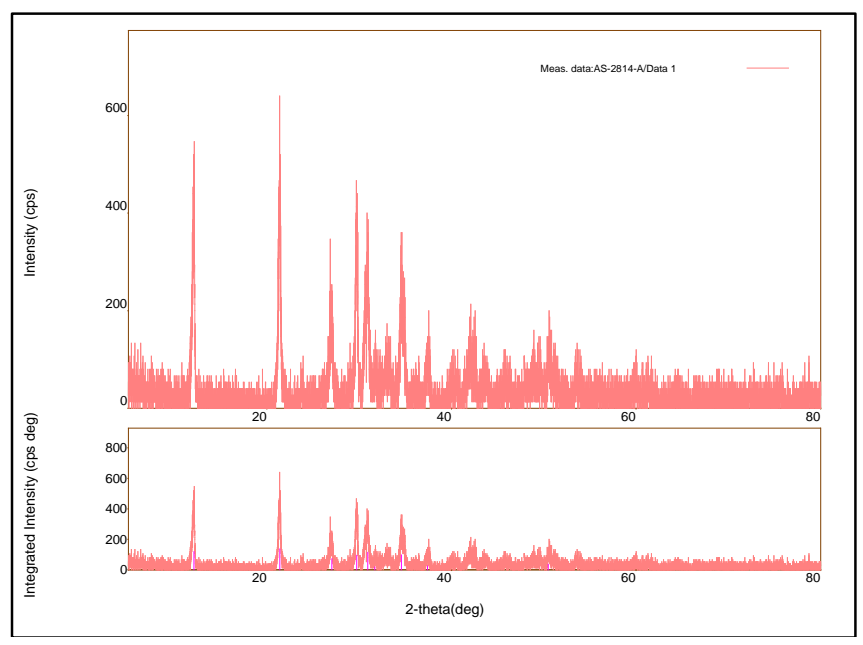

Fig 4: XRD pattern of nano -DCP

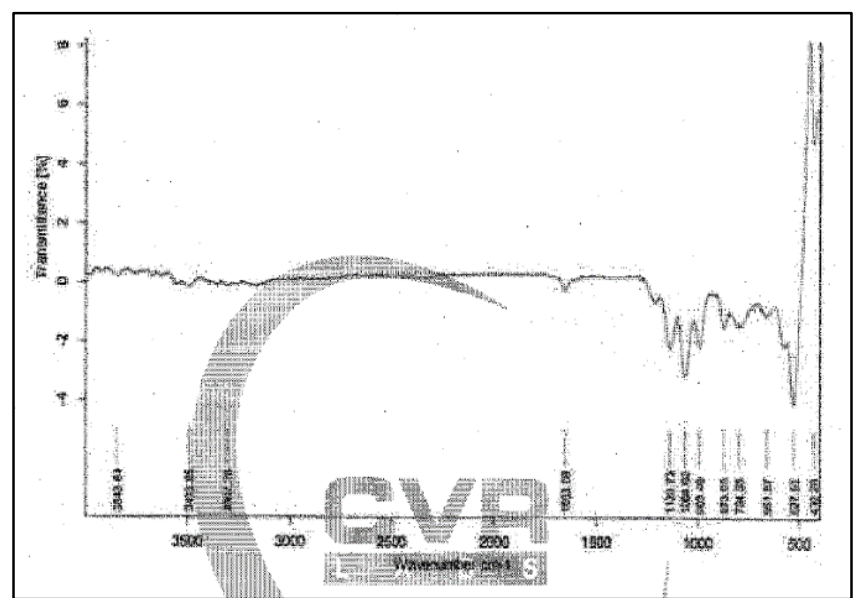

Fig 5: FTIR spectra of nano-DCP

\section{Fourier Transform Infrared Spectroscopy (FTIR)}

The typical FTIR spectra of synthesized nano particles of dicalcium phosphate showed well defined peaks between $4000-400 \mathrm{~cm}^{-1}$ (Fig. 5). The appearance of this peak was due to the presence of hydroxyl groups. The observed FTIR results confirmed that dicalcium phosphate nano particles can be synthesized without any significant impurities. Major peaks noticed in FTIR spectra were 3500, 3440, 1644, 1132, 1067,999 and $575 \mathrm{~cm}-1$ and these peaks corresponds to stretching and vibrational bending of $\mathrm{O}-\mathrm{H}$ stretching of residual free water, $\mathrm{O}-\mathrm{H}$ bending and rotation of residual free water, $\mathrm{P}-\mathrm{O}$ stretching and $\mathrm{O}-\mathrm{P}-\mathrm{O}(\mathrm{H})$ bending mode. Samy et al. (2015) ${ }^{[6]}$ observed similar findings for nano-DCP particles under FT-IR spectroscopy.

\section{Energy Dispersive Analysis by X-ray (EDAX)}

The result of EDAX (Fig.6) showed that nano dicalcium phosphate powder is composed of $23.22 \%$ Calcium and 17.67 $\%$ phosphorus and $\mathrm{Ca} / \mathrm{P}$ ratio of 1.31 . This finding was in concurrent to the reports of Ramesh (2014) ${ }^{[3]}$ who observed 23.12 and 16.93 per cent calcium and phosphorus content and $\mathrm{Ca} / \mathrm{P}$ ratio of 1.31 in nanoparticles prepared by ball milling method. In contrary to this Sagadevan and Dakshnamoorthy (2013) ${ }^{[18]}$ observed $\mathrm{Ca} / \mathrm{P}$ value of 1.68 .

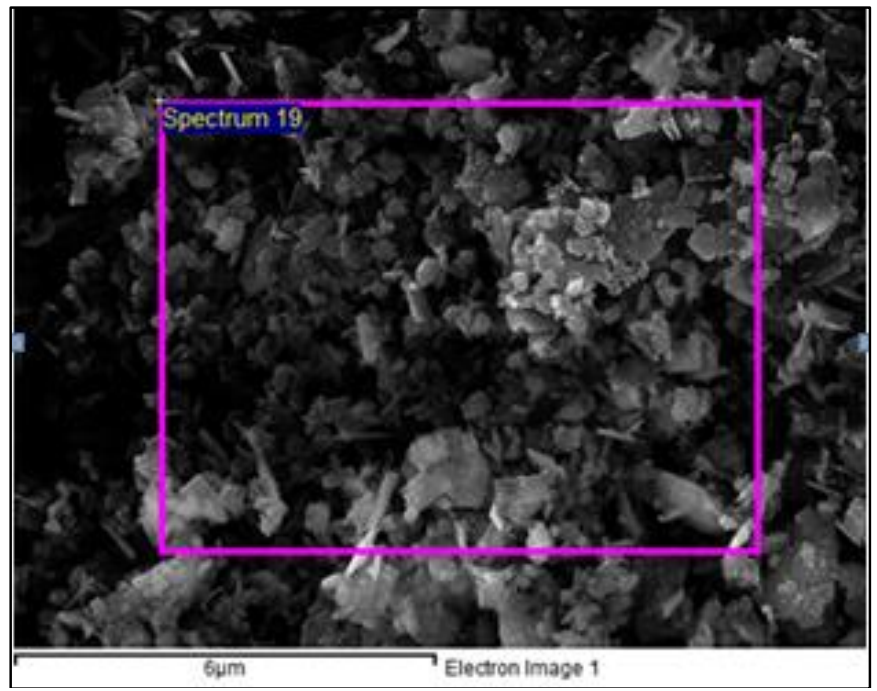

Fig 6: E-DAX spectra of nano-DCP

\section{Conclusion}

The present study indicates that the ball milling method can be successfully and effectively used for the synthesis of 47.5 $\mathrm{g} / \mathrm{hr}$ feed grade dicalcium phosphate (DCP) nanoparticles from $50 \mathrm{~g}$ of coarse DCP and it was found to be quick, easy to perform and economically feasible under field conditions. Based on its bio availability through biological trials it can be recommended in livestock and poultry feeding. It can also be supplemented to fast growing poultry species like broilers, turkey and meat type Japanese quail feed to prevent various leg and bone problems

\section{Acknowledgement}

The authors greatly acknowledged the Tamil Nadu Veterinary and Animal Sciences University, Chennai-51 for providing all infra structural facilities and research grant to carry out this work.

\section{Conflict of interest statement}

The author expresses no conflict of interest with any other individual or organisation regarding the information discussed in the manuscript.

\section{References}

1. Hassan HMA, Samy A, El-Sherbiny AE, Mohamed MA, Abd-Elsamee MO. Application of Nano-dicalcium Phosphate in Broiler Nutrition: Performance and Excreted Calcium and Phosphorus. Asian J Anim. Vet. Adv.2016; 11: 477-483.

2. Swain PS, Rajendran D, Rao SB, Dominic G. Preparation and effects of nano mineral particle feeding in livestock: A. review. Vet. World, 2015; 8(7):888-891

3. Ramesh J. Effect of nanomineral supplementation in TANUVAS smart mineral mixture on the performance of lambs. Ph.D thesis submitted Tamil Nadu Veterinary and Animal Science University, Chennai, 2014.

4. Huang J, Best SM, Bonfield W, Brooks RA, Rushton N, Jayasinghe SN, Edirisinghe MJ. In vitro assessment of the biological response to nano-sized hydroxyapatite. J Mater. Sci. Mater. Med. 2004; 15:441-445. 
5. Ota Y, Iwashita. Novel preparation method of hydroxyapatite fibres. J Am. Ceram. Soc. 1998; 81:16651668.

6. Samy A, Hassan HMA, El-Sherbiny AE, Abd-Elsamee MO, Mohamed MA. Characterization of nano dicalcium phosphate (ndcp) synthesized by sol-gel method. Int. J. Recent Scient. Res. 2015; 6(5):4091-4096.

7. Nakamura S, Tsobe T, Senna M. Hydroxyapatite nano sol prepared via a mechanochemical route. J Nanopart. Res. 2001; 3:57-61.

8. Sarig S, Kahana F. Rapid formation of nanocrystalline apatite. J. Crystal Growth. 2002; 25(2):237-239.

9. McCormick PG. Application of mechanical alloying to chemical refining. Mater. Trans., JIM, 1995; 36(2): 161169.

10. Cengiz B, Gokce Y, Yildiz N, Aktas Z, Calimli A. Synthesis and characterization of hydroxyapatite nanoparticles. Colloids and Surfaces A: Physicochem. Eng. Aspects, 2008; 322(1-3):29-33.

11. Azaroff LA. Elements of X-ray crystallography, McGraw-Hill, New york, 1968;38-42

12. Singh SP, Karmakar B. Mechanochemical synthesis of nano calcium silicate particles at room temperature. New Journal of Glass and Ceramics, (NJGC). 2011; 1(2):4952.

13. Nanocomposix. Zeta potential analysis of nanoparticles. Nanocomposix, 2012; 1(1):1-6

14. Welzel T, Radtke I, Meyer-zaika W, Heumann R, Epple M. Transfection of cells with custom made calcium phosphate nanoparticles coated with DNA. J Mater. Chem. 2004; 2213-2217.

15. Sokolova VV, Radtke I, Heumann R, Epple M. Effective transfection of cells with multi-shell calcium phosphateDNA nanoparticles. Biomaterials. 2006; 27(16):31473153.

16. Qing F, Wang Z, Hong Y, Liu M, Guo B, Luo H, Zhang v. Selective effects of hydroxyapatite nanoparticles on osteosarcoma cells and osteoblasts. J Mater. Sci. Mater. Med. 2012; 23(9):2245-2251.

17. Xiaochen Liu, Minzhi Zhao, Jingxiong Lu, Jian Ma, Jie Wei, W. Shicheng . Cell responses to two kinds of nanohydroxyapatite with different sizes and crystallinities. Int. J. Nanomedicine. 2012; 7:1239-1250.

18. Sagadevan S, Dakshnamoorthy A. Synthesis and characterization of nano-hydroxyapatite (n-HAP) using the wet chemical technique. Int. J Phys. Sci. 2013; 8(32):1639-1645. 